\title{
INFORMATION THEORETIC CAPACITY OF THE CELLULAR UPLINK - AVERAGE PATH LOSS APPROXIMATION
}

\author{
E. Katranaras, M. A. Imran and C. Tzaras \\ Centre for Communication Systems Research CCSR \\ University of Surrey, UK \\ Email: e.katranaras@surrey.ac.uk
}

\begin{abstract}
In this paper we investigate the information theoretic capacity of the uplink of a cellular system where all base station receivers jointly decode the received signals ("hyper-receiver"). Considering a distance depended power-law path loss and a more realistic Rician fading environment, we model a variable cell density network with geographically distributed user terminals. Multiple tiers of interference are considered and using an average path loss approximation model the analytical result for the per cell sum-rate capacity is found. We examine the various parameters that are affecting the capacity of the system. Especially the effect of the user distribution across the cells and the density of the cells in the cellular system is investigated. We validate the numerical solutions with Monte Carlo simulations for random fading realizations and we interpret the results for the real-world systems.
\end{abstract}

\section{INTRODUCTION}

The scientific field of information theory was successfully exploited to provide insight in the performance of cellular systems initially by Wyner [1]. The important result of maximum capacity when the Base Stations (BSs) cooperate to jointly decode the received signals led to further investigation of the problem. In [2], Wyner's simple yet tractable model was extended for flat fading environments. The assumptions of fixed cell density and interfering adjacent cells were tackled only recently by Letzepis [3] where Wyner's linear model was extended to incorporate distance dependent path loss and multiple-tier interference.

In this work the linear system considered in [3] is extended to the planar one. The Shannon-theoretic limits of uplink cellular systems are presented by overcoming the basic

The work reported in this paper has formed part of the "Fundamental Limits to Wireless Network Capacity" Elective Research Programme of the Virtual Centre of Excellence in Mobile \& Personal Communications, Mobile VCE, www.mobilevce.com. This research has been funded by the following Industrial Companies who are Members of Mobile VCE - BBC, BT, Huawei, Nokia, Nokia Siemens Networks, Nortel, Vodafone. Fully detailed technical reports on this research are available to staff from these Industrial Members of Mobile VCE. The authors would like to thank Prof. G. Caire and Prof. D. Tse for the fruitful discussions. limiting assumptions of Wyner's model. The effect of user distribution and of the cell density in the presence of powerlaw path loss is investigated in a general Rician flat fading environment. Multiple-tier interference is considered, providing insight in the role of cell density on the capacity of a centralised process considering Multiple Access Channel (MAC) cellular system. More specifically, it is shown that the capacity is a function of the received signal power. Considering that the magnitude of the received signal power for each transmitted signal depends on the path loss between the transmitter and the receiver and that the specific user distribution determines the values of the path loss coefficients for links between transmitters and receivers, one can say that the capacity depends on the user distribution. It is shown, finally, that the capacity can be approximated using the average path loss of different tiers of transmitters, for a given user distribution in a planar cellular network.

The rest of the paper is organised as follows. In section II a general view of the channel model considered is given. The path loss model is presented and the approximation method for three basic types of user distribution across the cells is further analysed. In section III the issue of the maximum information theoretic capacity is tackled with the mathematical tools of random matrix theory. The approach is taking into consideration a Rician fading environment where the phase randomness of the user signals leads to the maximum capacity. In section IV the various theoretical results are numerically presented along with Monte Carlo simulation results and we show a way of interpreting the results in the real-world systems. Finally, in section V we conclude the paper.

\section{ANALYSIS}

\subsection{Channel Model}

A planar hexagonal cellular array is assumed. Consider a network of $N^{2}$ cells and $K$ users in each cell. A BS at a cell will receive signals from all the users in the system, attenuated according to the employed power-law path loss and the existing fading environment. According to the above, the received signal at the base station antenna referring to cell $(m, n)$ is given 


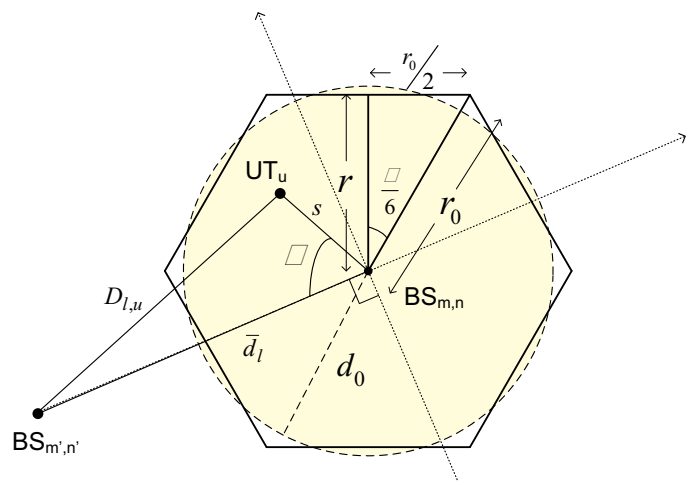

Fig. 1. A regular hexagonal cell with maximal radius of $r_{0}$, the circular equivalent of radius $d_{0}$ and the distance $D_{l, u}$ of a UT in a cell $(m, n)$ from a BS in a cell $(\dot{m}, n)$ at the $l^{\text {th }}$ tier of interference w.r.t. cell $(m, n)$.

by (with $N \rightarrow \infty$ ):

$$
\begin{aligned}
y_{m, n}= & \sum_{u=1}^{K}\left[\varsigma_{m, n, u} \cdot g_{m, n, u} \cdot x_{m, n, u}\right]+ \\
& \sum_{\dot{m}, \dot{n}=-\infty}^{\infty} \sum_{u=1}^{K}\left[\varsigma_{\dot{m}, \dot{n}, u} \cdot g_{\dot{m}, \dot{n}, u} \cdot x_{\dot{m}, \dot{n}, u}\right]+z_{m, n}
\end{aligned}
$$

where subscripts $m, n$ and $\dot{m}, \dot{n}$ (with $\dot{m}, \dot{n} \neq m, n$ ) identify the cell, in the 2D system, in which a user is located and $u$ identifies the user index. Furthermore, $\varsigma, g, x, y, z$ stand for path loss coefficients, fading coefficients, complex Gaussian inputs, complex Gaussian outputs and normalized (unit power) noise respectively. All the complex fading coefficients are normalized to unit power and when viewed as random complex processes are circularly symmetric i.i.d. Gaussian, strictly stationary and ergodic. It is assumed that each user has average power constraint $P$, i.e. $\mathbb{E}\left[x \cdot x^{*}\right] \leq P$. In accordance with Hanly's circular array model [4] a wrap-around toric model is adopted, where every cell has the same number of surrounding cells, so as to avoid the edge effects. Nevertheless, for large number of cells the edge effects do not affect the results [1].

\subsection{Path Loss Model}

A widely used model that maps the path loss and the distance in a power-law path loss environment is described by the variance profile function [3]:

$$
\varsigma_{\dot{m}, \dot{n}, u}=\left(1+D_{\dot{m}, \dot{n}, u}\right)^{-\eta / 2}
$$

where $D_{\dot{m}, \dot{n}, u}$ is a function describing the distance of user $u$ in cell $(\dot{m}, \dot{n})$ from the BS of cell $(m, n)$ and $\eta$ is the powerlaw path loss exponent.

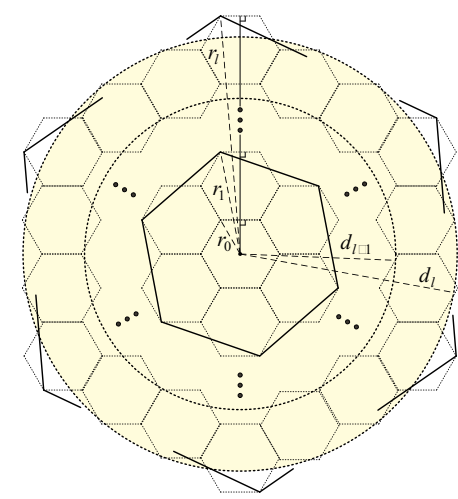

Fig. 2. Multiple tiers of interference around a cell. The irregular boundary of each tier can be represented by an equivalent regular hexagon and the latter by a circular boundary.

\subsection{Multiple Tier Interference}

Consider a regular hexagonal cell with its geometry given in Figure 1. In a planar cellular system we can have multiple tiers of interference around a cell as shown in Figure 2. The irregular boundary of each tier can be represented by an equivalent regular hexagon with its side length given by:

$$
r_{l}=\sqrt{[(2 l+1) \cdot r]^{2}+\left(\frac{r_{0}}{2}\right)^{2}}
$$

where $l$ stands for the $l^{\text {th }}$ tier of interference and $r, r_{0}$ are respectively the minimal and maximal radius of the hexagonal cell, as shown in Figure 1, with $r=r_{0} \cdot \cos \left(\frac{\pi}{6}\right)$. In general, a hexagonal type cell of side length $r_{l}$ can be approximated by a circular type one, as shown in the same figure, with radius given by:

$$
d_{l}=\frac{6}{\pi} \int_{0}^{\frac{\pi}{6}} \frac{r_{l}}{\cos \theta} \cos \left(\frac{\pi}{6}\right) d \theta
$$

According to the generic user distribution across the cells, for evaluating the capacity, it is useful to replace all the individual squared path loss coefficients of the users inside a cell $(m, n)$, that belongs to the $l^{t h}$ tier of interference with respect to the cell of interest $(m, n)$, with one mean squared path loss value $\overline{\varsigma_{l}^{2}}$. This mean squared path loss value can be estimated using the mean distance $\bar{d}_{l}$ of a BS in the $l^{t h}$ tier of interference from the BS of interest, which can be well approximated by the distance between the edges of the $l^{t h}$ and the $(l-1)^{t h}$ tier of interference from the BS of interest (see Fig. 2):

$$
\bar{d}_{l} \approx \frac{d_{l}+d_{l-1}}{2} .
$$

\subsection{User Distribution and Mean Squared Path Loss}

The mean squared path loss will be a function of the interference tier in which the cell belongs, as well as of the user distribution across the cell. For that purpose, we have to define 
the distance $D_{l, u}$ of a user in a cell in the $l^{t h}$ tier of interference from the BS of interest. With the help of Figure 1, one can prove that:

$$
D_{l, u}(\theta, s)=\sqrt{\left(s \cdot \sin \theta+\bar{d}_{l}\right)^{2}+(s \cdot \cos \theta)^{2}}
$$

where $s$ and $\theta$ define the location of a User Terminal (UT), distance and angle respectively, with respect to the BS in cell $(m, n)$ as shown in the same figure. Here, three different cases of user distribution are examined.

1. Uniform Distribution: In this case, the users are assumed to be uniformly distributed across the planar system. The mean squared path loss for the $K$ users in a cell which belongs in the $l^{\text {th }}$ tier of interference from the BS of interest is given by:

$$
\overline{\varsigma_{l-u n i}^{2}}=\frac{1}{\pi \cdot d_{0}{ }^{2}} \int_{0}^{d_{0}} \int_{-\pi}^{\pi} \frac{1^{2}}{\left(1+D_{l, u}(\theta, s)\right)^{\eta}} \cdot s \cdot d \theta d s
$$

Uniform distribution represents the most possible symmetric case and the most valid assumption when a large number of users is considered to be randomly placed across the system.

2. Truncated Cell-Centre Uniform Distribution: Here, the users are uniformly distributed around the centre of their cell and thus,

$$
\overline{\varsigma_{l-\text { centre }}^{2}}=\frac{1}{\pi \cdot \rho^{2}} \int_{0}^{\rho} \int_{-\pi}^{\pi} \frac{1^{2}}{\left(1+D_{l, u}(\theta, s)\right)^{\eta}} \cdot s \cdot d \theta d s
$$

where $\rho$ is the truncation factor that defines the radius around each BS in which the $K$ users are distributed with $\rho \ll 1$. For values of $\rho$ very close to zero (users are within the area of each BS), the mean squared path loss approaches to $\frac{1^{2}}{\left(1+\bar{d}_{l}\right)^{\eta}}$.

3. Truncated Cell-Edge Uniform Distribution: In this last case, the users are uniformly distributed on a disk segment close to the edge of their cell. We have,

$$
\overline{\varsigma_{l-e d g e}^{2}}=\frac{1}{\pi \cdot\left(d_{0}^{2}-\dot{\rho}^{2}\right)} \int_{\dot{\rho}}^{d_{0}} \int_{-\pi}^{\pi} \frac{1^{2}}{\left(1+D_{l, u}(\theta, s)\right)^{\eta}} \cdot s \cdot d \theta d s
$$

where $\rho$ is the truncation factor that defines the radius of the segment on which the users are distributed, with $d_{0}-\dot{\rho} \ll 1$. Note that for values of $\rho$ very close to $d_{0}$ (all users are very close to the edge of the cell), the mean squared path loss can be assumed to be given simply by $\frac{1}{\pi} \int_{-\frac{\pi}{2}}^{\frac{\pi}{2}} \frac{1}{\left(1+D_{l, u}(\theta, s)\right)^{\eta}} d \theta$.

\section{CAPACITY}

\subsection{General approach}

The output vector of all the received signals in the system can be rewritten based on channel equation (1) as:

$$
\mathbf{y}=\mathbf{H} \mathbf{x}+\mathbf{z}
$$

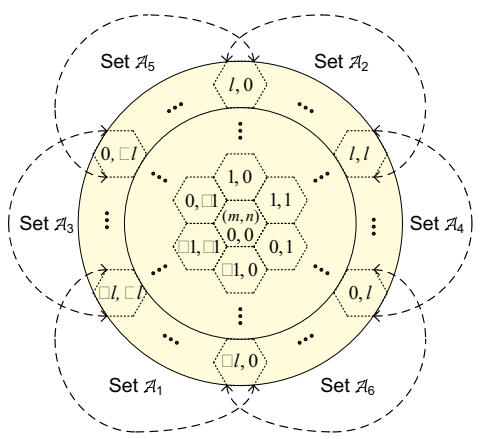

Fig. 3. The six sets defined, the concatenation of which describes which cells belong to the $l^{\text {th }}$ tier of interference of a cell $(m, n)$.

where $\mathbf{y}=\left[y_{1,1}, y_{1,2}, \ldots, y_{N, N}\right]^{T}$ is the $N^{2} \times 1$ received signal column vector, $\mathbf{x}=\left[\mathbf{x}_{1,1}{ }^{T}, \mathbf{x}_{1,2}{ }^{T}, \ldots, \mathbf{x}_{N, N}\right]^{T}$ is the concatenation of the transmitted signals of all the users to form a $N^{2} K \times 1$ column vector, with $\mathbf{x}_{m, n}=\left[x_{m, n, 1}, \ldots, x_{m, n, K}\right]$, denoting the concatenation of the transmitted signals from the $K$ users in cell $(m, n), \mathbf{z}$ is the $N^{2} \times 1$ column vector of noise and $\mathbf{H}$ is the overall $N^{2} \times N^{2} K$ system gain matrix. The overall system gain matrix is given by $\mathbf{H}=\boldsymbol{\Sigma} \odot \mathbf{G}$, where $\boldsymbol{\Sigma}$ is a deterministic $N^{2} \times N^{2} K$ matrix that contains all the path loss coefficients of the system channels and $\mathbf{G}$ is accordingly the $N^{2} \times N^{2} K$ matrix of all the fading coefficients. To create the above matrices, consider the representation of the system as a rectangular array as described in [1] and the raster scanning method that was used in [2] to define the order of the system output vector elements.

$\boldsymbol{\Sigma}$ is a Circulant matrix, in terms of its row-vector elements, which contain the path losses of all users in each cell. Regarding the $(m, n)^{t h}$ row of the matrix there can be defined six subsets to describe the cells that belong to the $l^{\text {th }}$ tier of interference and thus, the row blocks that contain the appropriate path losses (See Figure 3):

$$
\begin{aligned}
& \mathcal{A}_{1} \triangleq\{(m-l, n-l),(m-l, n-l+1), \cdots,(m-l, n)\} \\
& \mathcal{A}_{2} \triangleq\{(m+l, n),(m+l, n+1), \cdots,(m+l, n+l)\} \\
& \mathcal{A}_{3} \triangleq\{(m-l, n-l),(m-l+1, n-l), \cdots,(m, n-l)\} \\
& \mathcal{A}_{4} \triangleq\{(m, n+l),(m+1, n+l), \cdots,(m+l, n+l)\} \\
& \mathcal{A}_{5} \triangleq\{(m, n-l),(m+1, n-l+1), \cdots,(m+l, n)\} \\
& \mathcal{A}_{6} \triangleq\{(m-l, n),(m-l+1, n+1), \cdots,(m, n+l)\}
\end{aligned}
$$

Thus, the superset of these sets describes the cells belonging to the $l^{t h}$ tier of interference around the cell of interest.

The maximum per-cell capacity is achieved when all UTs are allowed to transmit all the time at their maximum power (WB scheme presented in [2]):

$$
C=\lim _{N \rightarrow \infty} \mathbb{E}\left[\frac{1}{N^{2}} \log _{2} \operatorname{det}\left(\boldsymbol{\Lambda}_{\mathbf{y}}\right)\right]
$$


where the expectation is taken over all the fading realizations and $\Lambda_{\mathbf{y}}$ is the covariance matrix of the output vector:

$$
\boldsymbol{\Lambda}_{\mathbf{y}}=P \cdot \mathbf{H H}^{\dagger}+\mathbf{I}_{N^{2} \times N^{2}}
$$

$\Lambda_{\mathbf{y}}$ is a large random matrix and Jensen's inequality with the law of large numbers can be used to provide us a tight upper bound for the capacity of the system [2]. Hence,

$$
C=\lim _{N \rightarrow \infty}\left(\frac{1}{N^{2}} \log _{2}\left(\operatorname{det} \mathbb{E}\left[\boldsymbol{\Lambda}_{\mathbf{y}}\right]\right)\right) \text { for } K \gg 1
$$

\subsection{Rician fading environment}

Assuming the fading coefficients normalized to unit power:

$$
\mathbb{E}\left[g_{m, n, u} \cdot\left(g_{m, n, u}\right)^{*}\right]=\mathbb{E}\left[\left(g_{m, n, u}\right)^{2}\right]=1
$$

Furthermore, considering the fading coefficients i.i.d., we have:

$$
\mathbb{E}\left[g_{m, n, u} \cdot\left(g_{m^{\prime}, n^{\prime}, u}\right)^{*}\right]=\left|m_{g}\right|^{2}
$$

where $m \neq m^{\prime}$ or $n \neq n^{\prime}$ and $m_{g}$ is the expected value of an individual fading coefficient.

Considering the random received phase on the specular path, a generalised model for the fading coefficients is [5]:

$$
g=\sqrt{\frac{\kappa}{\kappa+1}} \mathrm{e}^{j \phi}+\sqrt{\frac{1}{\kappa+1}} \mathcal{C N}(0,1)
$$

where $\mathbb{E}\left[g g^{*}\right]=1$ and $\kappa$ is used to define the ratio of the power in the Line of Sight (LOS) component and the other (non LOS) multipath components. The mean value of the product of two independent fading realizations is zero if the received signals on the specular path have uniformly distributed random phase offsets. Furthermore, the Rayleigh fading environment (no specular component) is, by its nature, a zero mean environment $\left(m_{g}=0\right)$.

Under these assumptions, the expectation of the covariance matrix of the conditioned output vector results in a Diagonal matrix. If we assume uniform user distribution, $\mathbb{E}\left[\boldsymbol{\Lambda}_{\mathbf{y}}\right]$ becomes (considering that there are always $6 \cdot l$ cells in the $l^{\text {th }}$ tier of interference):

$\mathbb{E}\left[\boldsymbol{\Lambda}_{\mathbf{y}-\text { uni }}\right]=\operatorname{diag}\left[1+K P \cdot\left(\overline{\varsigma_{0-u n i}^{2}}+\sum_{l=1}^{\infty} 6 \cdot l \cdot \overline{\varsigma_{l-u n i}^{2}}\right)\right]$

where $\overline{\varsigma_{0-u n i}^{2}}$ denotes the mean squared path loss for the users inside the cell of interest. Taking into consideration equations (14) and (18), the asymptotic expression of the maximum percell capacity for any finite number of users $K$ is given by:

$$
C_{R-\text { uni }}=\log \left[1+K P \cdot\left(\overline{\varsigma_{0-u n i}^{2}}+\sum_{l=1}^{\infty} 6 \cdot l \cdot \overline{\varsigma_{l-u n i}^{2}}\right)\right]
$$

which is a strict upper bound for the capacity in the system. Following the same procedure for the other two types of distribution, we obtain similar expressions for the capacity.

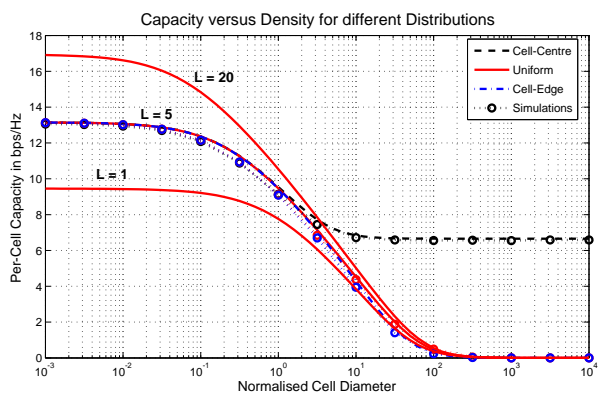

Fig. 4. Capacity versus the normalised cell diameter for different distributions and number of interfering tiers $L$ around each cell. Transmit SNR $=20 \mathrm{~dB}$ per cell, $\eta=2$.

\section{RESULTS}

In the following, some interesting results on the information theoretic capacity of planar cellular systems are presented. We note also that, in Figures 4 and 5, the extreme only cases of the different distributions ape presented, e.g. for the truncated cell-centre distribution, $\rho$ was considered to be equal to zero (all the users at the centre of their cell), while for the truncated cell-edge distribution, $\rho$ was considered to be equal to $d_{0}$ (all the users at the edge of their cell).

In Figure 4 the theoretical results have been verified by running Monte Carlo simulations to generate random fading coefficients and finding the long term average capacity:

$$
C_{\text {sim }}=\frac{1}{N^{2}} \mathbb{E}\left[\log _{2} \operatorname{det}\left(\mathbf{I}_{N^{2} \times N^{2}}+P \cdot \mathbf{H H}^{\dagger}\right)\right]
$$

The per-cell capacity is plotted for the different cases of user distribution against the normalised cell diameter. The small difference between the simulations and the analytical results can be attributed to the fact that for finding $C_{\text {sim }}$ a finite system of 91 cells was considered instead of a wrap-around toric one. It can be seen that for low normalised cell diameter the capacities for all distributions coincide and reach the maximum possible value. On the other hand, as the size of the cells grows large, the capacity decreases. Specifically, for uniform and truncated cell-edge user distributions, capacity tends to zero, as it was expected, with the former having a smoother decrease after a specific cell size. For truncated cell-centre distribution, capacity decreases with the size of the cell and reaches to a specific non-zero value. This is due to the fact that the users of the cell of interest will always be close to their base stations no matter how large the size of the cell will be. Note that, in all cases, uniform and truncated cell-edge distributions provide capacities very close to each other. Furthermore, the behaviour of the capacity is examined while the number of interfering tiers of cells changes. It can be seen that for high values of normalised cell diameter the number of interfering tiers has no significant role on the capacity. As the system becomes more dense, the capacity increases with 


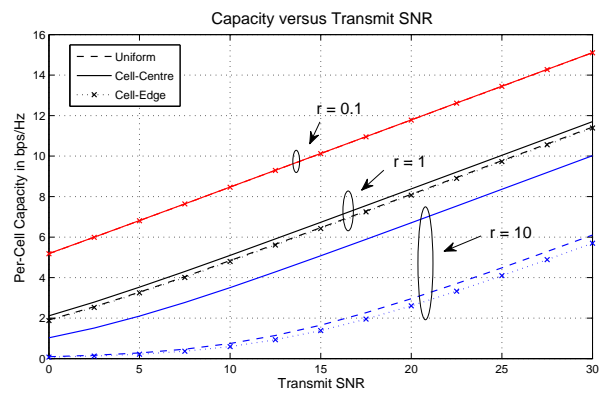

Fig. 5. Capacity versus Transmit SNR per cell for different BS densities and user distributions. $L=5$ and $\eta=2$.

the number of the interfering tiers but with a decreasing rate.

In Figure 5, the capacity is plotted versus the transmit per-cell SNR for different system densities and user distributions. We note that capacity increases with the transmit per-cell power. For dense and normal cell density systems the capacity does not change significantly for different user distributions. On the other hand, for low cell density systems the capacity with truncated cell-centre distribution is significantly higher than for the other two distribution cases.

We apply the analysis to a real-world scenario. Consider a scenario [6] where cells have radii of $100 \mathrm{~m}-3 \mathrm{~km}$, the path loss at a reference distance of $1 \mathrm{~m}$ is $-38 \mathrm{~dB}$ (for a carrier frequency of $1.9 \mathrm{GHz}$ ) and the path loss exponent is 2 or 3.5. The UTs are uniformly distributed over cells with user transmit power constraint of 100-200 $\mathrm{mW}$ and thermal noise density of -169 $\mathrm{dBm} / \mathrm{Hz}$ with channel bandwidth of $5 \mathrm{MHz}$. A maximum of 5 tiers of interfering cells is assumed for every cell. Random received phases are assumed to plot the capacity in Figure 6 against the Rise over Thermal (RoT). When plotting the capacity against the RoT one can say that a more general view of the capacity behaviour is obtained. The different parameters specify only the operating area for the capacity of our system but they do not change the behaviour of the capacity which follows a Shannon-like formula:

$$
C=\log _{2}[1+\mathrm{RoT}]
$$

We note that the capacity increases drastically for a relatively low path loss exponent and when the cell size decreases. Furthermore, it is shown that increasing number of users increases the capacity but with a decreasing rate. We can also observe that an increase in the transmitting user power from 100 to $200 \mathrm{~mW}$ doesn't have a significant effect on the capacity.

\section{CONCLUSION}

We investigate the capacity of the planar cellular uplink channel. An average path loss approximation model was presented for the analysis of a system where every base station receives signals from the same cell and the surrounding cell users. The

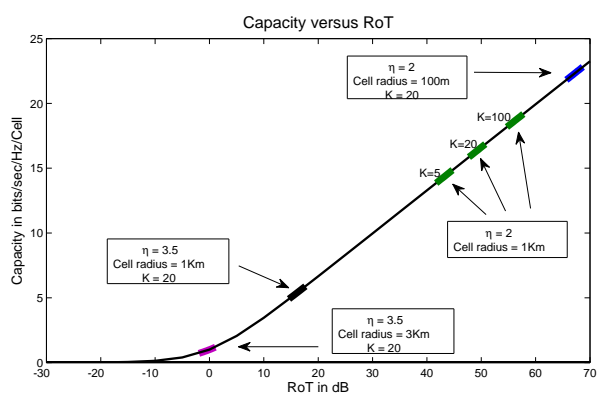

Fig. 6. Capacity versus RoT for different path loss exponents $(\eta=2,3.5)$ and cell radius $(100 \mathrm{~m}, 1 \mathrm{Km}, 3 \mathrm{Km})$. Uniformly distributed users with transmit power varying between $100-$ $200 \mathrm{~mW}, L=5$.

size of the cells and hence the cell density is modelled as a variable. Assuming a joint decoder at the base stations ("hyper receiver" scheme) a tight upper bound, for the maximum per cell sum-rate capacity, is provided. Various parameters of a practical system that affect the per-cell capacity are identified and analysed. Specifically, these parameters are: (1). the user distribution across the cell, (2). the cell size , (3). the path loss exponent, (4). the transmit power constraint of the users, and (5). the number of users per cell. These parameters have an effect on a unified parameter - the Rise over Thermal (RoT) at each base station which is shown to directly control the information theoretic capacity of the system.

\section{REFERENCES}

[1] A.D. Wyner, "Shannon-theoretic approach to a gaussian cellular multiple-access channel," Information Theory, IEEE Transactions on, vol. 40, no. 6, pp. 1713-1727, Nov. 1994.

[2] O. Somekh and S. Shamai, "Shannon-theoretic approach to a gaussian cellular multiple-access channel with fading," Information Theory, IEEE Transactions on, vol. 46, no. 4, pp. 1401-1425, July 2000.

[3] N. Letzepis and A. Grant, "Information capacity of multiple spot beam satellite channels," in Communications Theory Workshop, 2005. Proceedings. 6th Australian, 24 Feb. 2005, pp. 168-174.

[4] S. V. Hanly and P. A. Whiting, "Information-theoretic capacity of milti-receiver networks," Tellecommun. Syst., vol. Vol. 1, pp. pp. 1-42, 1993.

[5] D. Tse and P. Viswanath, Fundamentals of Wireless Communications, University Press, Cambridge, 2005.

[6] Theodore S. Rappaport, Ed., Wireless Communications: Principles and Practice, Prentice-Hall, Inc., Upper Saddle River, NJ, USA, 1995. 